\title{
Saúde mental de crianças e adolescentes vítimas de violência: uma revisão crítica da literatura*
}

\author{
Mental health of children and teens victims of \\ violence: a critical review of the literature
}

\author{
Kaíla da Silva Bontempo ${ }^{1}$, Andrea Ruzzi Pereira ${ }^{2}$
}

BONTEMPO, K. S.; PEREIRA, A. R. Saúde mental de crianças e adolescentes vítimas de violência: uma revisão crítica da literatura. Rev. Ter. Ocup. Univ. São Paulo, v. 23, n. 2, p. 130-136, maio/ ago. 2012 .

RESUMO: Trata-se de uma revisão crítica da literatura que objetivou verificar e analisar a produção bibliográfica em periódicos brasileiros sobre as consequências da violência na saúde mental de crianças e adolescentes vítimas de violência. Efetuou-se a busca dos artigos publicados entre janeiro de 2002 e dezembro 2010 nas bases de dados eletrônicas Lilacs e Scielo. Os dados foram distribuídos e analisados em quatro agrupamentos: artigos pesquisados, a abordagem metodológica, os diversos tipos de violência e seus fatores de risco, e a violência e suas consequências para saúde mental de crianças e adolescentes. Foram encontrados 15 artigos. Obteve-se que maioria dos artigos tinha como população alvo adolescentes e que os estudos foram realizados prioritariamente em escolas e serviços de saúde; que as consequências da violência para a saúde mental infanto-juvenil foram abordadas nos artigos conforme o instrumento utilizado por eles para a coleta de dados ou de acordo com o comportamento dessa população; que os sintomas mais citados pelos autores são comportamento depressivo, ansiedade e condutas anti-sociais. Percebe-se a falta de pesquisas que relatem intervenções realizadas para prevenir ou tratar as consequências da violência para a saúde mental dessa população.

DESCRITORES: Adolescente, Criança, Saúde Mental, Violência

\footnotetext{
* Resultado do Trabalho de Conclusão de Curso (TCC) do Curso de Terapia Ocupacional da Universidade Federal do Triângulo Mineiro (UFTM).

1. Graduanda do Curso de Terapia Ocupacional da Universidade Federal do Triângulo Mineiro - UFTM.

2. Prof ${ }^{a}$ Assistente do Curso de Terapia Ocupacional da Universidade Federal do Triângulo Mineiro - UFTM.

Endereço para correspondência: Universidade Federal do Triângulo Mineiro. Unidade Centro Educacional (CE). Curso de Terapia

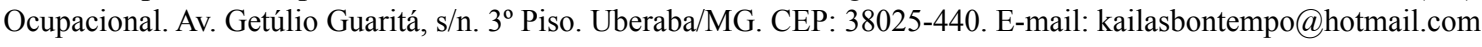




\section{INTRODUÇÃO}

violência, por ser um fenômeno complexo,
é definida como: ou em ameaça, contra si próprio, contra outra pessoa, ou contra um grupo ou uma comunidade, que resulte ou tenha grande possibilidade de resultar em lesão, morte, dano psicológico, deficiência de desenvolvimento ou privação (WHO, 1996 apud KRUG et. al., 2002, p. 4).

Em 2002, a Organização Mundial de Saúde Publicou o Relatório Mundial sobre Violência e Saúde. Este documento contribui de várias maneiras para o conhecimento, a identificação, a prevenção e o enfrentamento da violência, pois descreve desde os tipos de violência e seus fatores de risco até a forma de enfrentá-la nos diferentes níveis: local, nacional e internacional (KRUG et al, 2002).

O Relatório Mundial classifica a violência quanto à natureza dos atos como física, psicológica e sexual; por privação ou negligência; e de acordo com quem a comete como violência contra si mesmo, que inclui comportamento suicida e autodestrutivo, violência interpessoal subdividida em violência familiar ou íntima e violência comunitária, violência coletiva cometida contra um grupo de pessoas, geralmente com fins políticos, econômicos ou sociais (KRUG et al., 2002, p. 5). Para discutirmos como a violência tem sido abordada em pesquisas brasileiras, tomaremos como referência esse documento e suas proposições.

A violência de forma geral, principalmente contra crianças e adolescentes, é considerada um problema mundial de saúde pública. Dados do Ministério da Saúde apontam que esta pode ocasionar problemas emocionais, psicológicos, sociais e cognitivos durante esta fase da vida ou nas posteriores (BRASIL, 2008). Em relação à saúde mental, algumas das consequências podem ser: ansiedade, transtornos depressivos, alucinações, baixo desempenho na escola e tarefas de casa, alterações de memória, comportamento agressivo, defensivos ou violentos, retraimento, e até tentativas de suicídio (AVANCI et al., 2009; BRASIL, 2008; KRUG et al., 2002).

Neste sentido, busca-se conhecer como as pesquisas têm abordado a questão da violência e suas consequências para a saúde mental de crianças e adolescentes. Portanto, os objetivos deste estudo foram identificar e analisar, em periódicos brasileiros, a produção bibliográfica sobre as consequências na saúde mental de crianças e adolescentes vítimas de violência, no período de 2002 a 2010, a partir da publicação do Relatório Mundial Sobre Violência e Saúde.

\section{METODOLOGIA}

Neste estudo optou-se por uma revisão crítica que apresenta, analisa e sintetiza o material a partir de fontes diversas, indo além da simples descrição dos artigos, resultando em uma síntese de modelos existentes (GRANT; BOOTHT, 2009).

Efetuou-se a busca de artigos nas bases de dados eletrônicas Lilacs e Scielo. Foram incluídos artigos que tomaram como sujeitos crianças e adolescentes de zero a 18 anos incompletos, publicados em periódicos brasileiros entre janeiro de 2002 a dezembro de 2010 e disponíveis na íntegra. Foram excluídos da pesquisa artigos de revisão bibliográfica ou de literatura.

Para a busca dos artigos os descritores foram separados em três categorias, utilizados em combinações 3 a 3. As categorias foram: saúde mental; violência, incluindo as palavras violência e abuso; infância e adolescência, incluindo criança(s), neonato(s), infância, desenvolvimento infantil, adolescente(s), adolescência e puberdade. A pré-seleção dos artigos foi realizada no primeiro trimestre de 2011, de forma independente pelas pesquisadoras através da leitura dos títulos e dos resumos. Quando os resumos não traziam dados suficientes ou havia discrepâncias entre as seleções de cada pesquisadora, era realizada a leitura dos artigos na íntegra para verificar a inclusão ou não do artigo.

Os artigos selecionados foram lidos e submetidos a uma avaliação qualitativa, que consistiu na leitura dos artigos na íntegra, sendo todo material fichado, analisado, comparado e avaliado quanto a sua contribuição para o objetivo deste estudo. As informações foram sistematizadas em um instrumento que contemplou os seguintes itens: autores, ano de publicação, população alvo, local, objetivos, delineamento metodológico e resultados dos estudos.

A análise dos dados foi baseada na análise de conteúdo proposta por Bardin (2010) utilizando a técnica de análise categorial, dividindo os achados em quatro agrupamentos: artigos pesquisados, abordagem metodológica dos artigos pesquisados, os diversos tipos de violência e seus fatores de risco, e a violência e suas consequências para saúde mental de crianças e adolescentes.

\section{RESULTADOS}

\section{Artigos pesquisados}

Foram encontrados 32 artigos, sendo que desses 17 foram excluídos após a leitura na íntegra por não responderem aos critérios de inclusão, o que resultou em 15 artigos para análise.

Os estudos foram realizados e publicados, em sua maior parte, nos estados Rio de Janeiro (6) e São Paulo (4). Verifica-se que apenas um dos estudos foi realizado na região Nordeste do Brasil e nenhum na região Norte. 
Entre as revistas destacam a Revista Ciência e Saúde Coletiva e a Revista de Saúde Pública, com, respectivamente, quatro e três estudos. Ressalta-se que ambas as revistas priorizam contribuições científicas relacionadas à saúde pública na qual a violência é incluída por sua característica plurifacetada.

Tabela 1 - Caracterização e desenho dos artigos pesquisados

\begin{tabular}{|c|c|c|c|c|c|c|c|c|}
\hline $\begin{array}{l}\text { Identifi- } \\
\text { cação do } \\
\text { artigo }\end{array}$ & Autores & Ano & $\begin{array}{l}\text { Estado de } \\
\text { produção } \\
\text { do artigo }\end{array}$ & Revista & $\begin{array}{l}\text { População } \\
\text { abordada no } \\
\text { artigo }\end{array}$ & Local & $\begin{array}{c}\text { Instrumentos } \\
\text { padronizados } \\
\text { utilizados }\end{array}$ & Abordagem \\
\hline N1 & $\begin{array}{l}\text { Boarati, } \\
\text { M.C.B; et. al. }\end{array}$ & 2009 & SP & $\begin{array}{c}\text { Brasileira de } \\
\text { Crescimento e } \\
\text { Desenvolvimento } \\
\text { Humano }\end{array}$ & Crianças & Ambulatório & Nenhum & Qualitativa \\
\hline N2 & $\begin{array}{l}\text { Avanci, J; } \\
\text { et. al. }\end{array}$ & 2009 & RJ & $\begin{array}{l}\text { Ciência e Saúde } \\
\text { Coletiva }\end{array}$ & Crianças & Escola & $\begin{array}{l}\text { CBCL } \\
\text { ETC }\end{array}$ & Quantitativo \\
\hline N3 & $\begin{array}{l}\text { Souza, M. K. } \\
\text { B; et. al. }\end{array}$ & 2009 & BA & $\begin{array}{c}\text { Ciência e Saúde } \\
\text { Coletiva }\end{array}$ & Adolescentes & $\begin{array}{l}\text { Rede Pública } \\
\text { de Saúde }\end{array}$ & Nenhum & Qualitativa \\
\hline N4 & $\begin{array}{l}\text { Tanaka, O. U; } \\
\text { et. al. }\end{array}$ & 2009 & SP & $\begin{array}{l}\text { Ciência e Saúde } \\
\text { Coletiva }\end{array}$ & Crianças & UBS & $\begin{array}{l}\text { CBCL } \\
\text { SDQ }\end{array}$ & $\begin{array}{c}\text { Quantitativa e } \\
\text { Qualitativa }\end{array}$ \\
\hline N5 & $\begin{array}{l}\text { Oliveira, A. } \\
\mathrm{S} ; \text { et. al. }\end{array}$ & 2006 & GO & $\begin{array}{l}\text { Eletrônica de } \\
\text { Enfermagem }\end{array}$ & Adolescentes & Escola & Nenhum & Qualitativa \\
\hline N6 & $\begin{array}{l}\text { Werneck, G. } \\
\text { L; et. al. }\end{array}$ & 2006 & RJ & $\begin{array}{l}\text { Cadernos de } \\
\text { Saúde Pública }\end{array}$ & Adolescentes & Hospital & Nenhum & Quantitativo \\
\hline N7 & $\begin{array}{l}\text { Paula, C. S; } \\
\text { et. al. }\end{array}$ & 2008 & SP & Saúde Pública & Adolescentes & Escola & SDQ & Quantitativo \\
\hline N8 & $\begin{array}{l}\text { Ruzany, M. } \\
\mathrm{H} \text {; et. al. }\end{array}$ & 2010 & RJ & Pediatria Moderna & Adolescentes & Comunidades & Nenhum & Quantitativo \\
\hline N9 & $\begin{array}{l}\text { Ximenes, L. } \\
\text { F; et. al. }\end{array}$ & 2009 & RJ & $\begin{array}{l}\text { Ciência e Saúde } \\
\text { Coletiva }\end{array}$ & Crianças & Escolas & $\begin{array}{c}\text { CTS } \\
\text { WISC III } \\
\text { CBCL }\end{array}$ & Quantitativo \\
\hline N10 & $\begin{array}{l}\text { Avanci, J; } \\
\text { et. al. }\end{array}$ & 2007 & RJ & $\begin{array}{l}\text { Psicologia Teoria } \\
\text { e Pesquisa }\end{array}$ & Adolescentes & Escolas & SRQ 20 & Quantitativo \\
\hline N11 & $\begin{array}{l}\text { Benetti, S. P. } \\
\text { C; et. al. }\end{array}$ & 2010 & RS & $\begin{array}{l}\text { Universidade de } \\
\text { São Francisco }\end{array}$ & Adolescentes & Escolas & $\begin{array}{c}\text { IEP } \\
\text { IEEA } \\
\text { QTECVC } \\
\text { CBCL }\end{array}$ & Quantitativa \\
\hline N12 & $\begin{array}{l}\text { Arpini, D. M; } \\
\text { et. al. }\end{array}$ & 2008 & RS & $\begin{array}{c}\text { Psicologia em } \\
\text { Revista }\end{array}$ & $\begin{array}{c}\text { Crianças e } \\
\text { Adolescentes }\end{array}$ & $\begin{array}{l}\text { Escolas e } \\
\text { UBS }\end{array}$ & Nenhum & Qualitativa \\
\hline N13 & $\begin{array}{l}\text { Assis, S. G; } \\
\text { et. al. }\end{array}$ & 2009 & RJ & Saúde Pública & Crianças & Escolas & CBCL & Quantitativo \\
\hline N14 & $\begin{array}{l}\text { Marcondes } \\
\text { Filho, W; } \\
\text { et. al. }\end{array}$ & 2002 & PR & $\begin{array}{c}\text { Adolescência } \\
\text { Latino Americana }\end{array}$ & Adolescentes & Hospital & $\begin{array}{l}\text { DSM-IVTM } \\
\text { CAGE }\end{array}$ & Qualitativa \\
\hline N15 & $\begin{array}{l}\text { Vitolo, Y; } \\
\text { et. al. }\end{array}$ & 2005 & SP & Saúde Pública & Crianças & Escolas & $\begin{array}{c}\text { SDQ } \\
\text { SRQ 20 }\end{array}$ & Quantitativo \\
\hline
\end{tabular}

UBS= Unidade Básica de Saúde; $\mathrm{CBCL}=$ Child Behavior Checklist; $\mathrm{ETC}=$ Escala Tática de Conlfitos $\mathrm{SDQ}=\mathrm{Strengths}$ and Difficulties Questionnaire; CTS= Escala Tática de Conflitos; WISC III= Escala de Inteligência Wechsler para criança; SRQ $20=$ Self Reported Questionnarie; IEP= Inventário de Estilos Parientais; IEEA= Inventário de Eventos Estressores na Adolescência; QTECVC = Questionário Triagem da Exposição de Crianças à Violência na Comunidade; DSM- IVTM = Manual Diagnóstico e Estatístico de Transtornos Mentais; CAGE= acrônimo referente às suas quatro perguntas- Cut down, Annoyed by criticism, Guilty e Eye-opener. 


\section{A abordagem metodológica dos artigos pesquisados}

Ao analisar-se o desenho do estudo dos artigos encontrados, destacou-se a população abordada, o local, os instrumentos padronizados utilizados e a abordagem da pesquisa.

Conforme a tabela 1, oito artigos pesquisados escolheram os adolescentes como população alvo e a escola como o local para a coleta dos dados. Esta coleta foi realizada por nove artigos através de instrumentos padronizados, destacando principalmente o Child Behavior Checklist -CBCL, Strengths and Difficulties Questionnaire - SDQ e o Self Reported Questionnaire - SRQ 20.

Em relação ao tipo de abordagem da pesquisa oito estudos são quantitativos, cinco são qualitativos e um quantitativo-qualitativo.

\section{Os diversos tipos de violência e seus fatores de risco}

De acordo com a Tabela 2 verifica-se que 13 artigos estudados abordam principalmente a violência interpessoal e apenas dois deles a violência contra si mesmo. Quanto à natureza do ato, a violência física associada à violência psicológica é a mais citada (5), embora todos os quatro tipos física, psicológica, sexual e por privação ou negligência, sejam explorados por pelo menos um dos artigos, separados ou conjuntamente. O suicídio foi abordado em dois artigos (N6 e N14).

\section{A violência e suas consequências para saúde mental de crianças e adolescentes}

Os artigos pesquisados abordam alguns sintomas depressivos e ansiosos como consequências da violência para a saúde mental de crianças e adolescentes. Classificam esses sintomas conforme o instrumento que utilizaram para a coleta de dados ou de acordo com o comportamento apresentado por essas crianças e adolescentes.

Os artigos que usaram a CBCL como instrumento de coleta dividiram os sintomas em internalizantes, e externalizantes (ASSIS; AVANCI; OLIVEIRA, 2009; AVANCI et. al., 2009; BENETTI et al., 2010; TANAKA; RIBEIRO, 2009). Os artigos que utilizaram a escala SRQ 20 como instrumento classificaram os sintomas em psicossomáticos, depressivos e ansiosos (AVANCI et al., 2007; VITOLO et al., 2005). E os que usaram o SDQ apresentam como consequência ansiedade e/ou depressão, problemas de conduta, hiperatividade/déficit de atenção, problema de relacionamento e comportamento social positivo (PAULA, et al., 2008; TANAKA e RIBEIRO; 2009; VITOLO et al., 2005).
Independente dos instrumentos utilizados, os sintomas mais citados pelos autores foram comportamento depressivo, ansiedade e condutas antissociais como comportamento desafiador, violento/agressivo ou disruptivo e delinquência. O Transtorno de Estresse Pós-Traumático também foi abordado por dois artigos e os sintomas que o caracterizam são separados em três grandes grupos: revivência do evento traumático, esquiva persistente de estímulos associados ao evento e excitabilidade aumentada (XIMENES; OLIVEIRA; ASSIS, 2009).

Os artigos que não utilizaram instrumento padronizado na coleta de dados apresentaram como consequências à saúde mental os mesmos sintomas identificados pelos instrumentos (Tabela 2).

\section{DISCUSSÃO}

A partir dos dados apresentados na tabela 1 é possível observar que a maior parte das pesquisas se concentraram nas grandes metrópoles o que impossibilita a identificação e comparação dos tipos de violência e suas possíveis consequências para crianças e adolescentes nas outras regiões do país.

Houve também, nos artigos pesquisados, uma preferência em relação a população alvo e o local para a realização da coleta de dados, sendo escolhidos os adolescentes e a escola, respectivamente.

Em dois artigos os autores destacam que na adolescência os fatores e sintomas relacionados à saúde mental se sobressaem, quando analisados, devido às próprias características desta fase da vida, como a instabilidade emocional, a construção da autoestima, a inserção em grupos sociais, a busca pela independência e valorização pela sociedade (AVANCI et. al., 2007; OLIVEIRA; ANTONIO, 2006).

Quanto ao local para a coleta de dados, Avanci et al. (2007, p. 288) discorre que:

A abordagem de populações escolares no ambiente de ensino constitui uma alternativa bastante plausível, seja pelo alcance de maior tamanho amostral, possibilitando maior poder estatístico nas análises realizadas, seja pela relativa facilidade de acesso, auxiliando a operacionalização da coleta dos dados; ou mesmo por ser um local privilegiado, no qual boa parte dos adolescentes frequenta, coadunando experiências e realidades distintas.

Sendo assim, a escola pode ser um ambiente tanto para identificação e reconhecimento das situações de violências e as possíveis consequências para saúde mental quanto para o desenvolvimento de ações de enfrentamento e prevenção da violência (BENETTI et al., 2010). 
Tabela 2 - Caracterização dos tipos de violência abordados na amostra e consequências para saúde mental abordadas nos artigos da pesquisa

\begin{tabular}{|c|c|c|c|}
\hline Identificação & $\begin{array}{l}\text { Tipo de Violência: } \\
\text { Quem Comete }\end{array}$ & Tipo de Violência: Natureza dos Atos & Sintomas \\
\hline N1 & Interpessoal & Sexual & Comportamento desafiador e agressivo \\
\hline $\mathrm{N} 2$ & Interpessoal & Física e Psicológica & $\begin{array}{c}\text { Comportamento depressivo } \\
\text { Baixa auto-estima } \\
\text { Ansiedade } \\
\text { Comportamento disruptivo }\end{array}$ \\
\hline N3 & Interpessoal & $\begin{array}{c}\text { Física; Psicológica; Sexual; e por Privação } \\
\text { ou Negligência }\end{array}$ & Comportamentos violentos e anti-sociais \\
\hline N4 & Interpessoal & Física e Psicológica & $\begin{array}{c}\text { Psicossomáticos } \\
\text { Agitação } \\
\text { Insônia } \\
\text { Dificuldades na escola } \\
\text { Baixa auto-estima }\end{array}$ \\
\hline N5 & Interpessoal & Psicológica & $\begin{array}{l}\text { Ansiedade } \\
\text { Agressividade }\end{array}$ \\
\hline N6 & Contra si mesmo & -- & -- \\
\hline N7 & Interpessoal & Física & $\begin{array}{c}\text { Ansiedade } \\
\text { Depressão } \\
\text { Isolamento social } \\
\text { Problemas de conduta e deliquência }\end{array}$ \\
\hline $\mathrm{N} 8$ & Interpessoal & Negligência e Física & $\begin{array}{c}\text { Distúrbios de personalidade } \\
\text { Comportamentos Violentos e anti-sociais } \\
\text { Dependência Química } \\
\text { Problemas de aprendizado } \\
\text { Depressão }\end{array}$ \\
\hline N9 & Interpessoal & Física & $\begin{array}{c}\text { Ansiedade } \\
\text { TEPT } \\
\text { Depressão } \\
\text { Agressividade }\end{array}$ \\
\hline N10 & Interpessoal & Psicológica & $\begin{array}{c}\text { TPM } \\
\text { Psicossomáticos } \\
\text { Depressivos } \\
\text { Ansiosos }\end{array}$ \\
\hline N11 & Interpessoal & Física e Psicológica & $\begin{array}{c}\text { Comportamento agressivo e disruptivo } \\
\text { Depressão } \\
\text { Ansiedade } \\
\text { Retraimento } \\
\text { Psicossomáticos }\end{array}$ \\
\hline $\mathrm{N} 12$ & Interpessoal & Física e Psicológica & $\begin{array}{l}\text { Mudanças de comportamento } \\
\text { Dificuldades de aprendizagem }\end{array}$ \\
\hline N13 & Interpessoal & Privação e Física & $\begin{array}{c}\text { Depressão } \\
\text { Ansiedade } \\
\text { Agressividade } \\
\text { Dificuldade de atenção }\end{array}$ \\
\hline N14 & Contra si mesmo & -- & -- \\
\hline N15 & Interpessoal & Física e Psicológica & $\begin{array}{c}\text { Ansiedade } \\
\text { Depressão } \\
\text { Problemas de conduta } \\
\text { Hiperatividade/ déficit de atenção } \\
\text { Problemas de relacionamento com colegas }\end{array}$ \\
\hline
\end{tabular}

Para embasar a sua coleta de dados a maioria dos autores optaram por utilizar instrumentos padronizados, o que reflete, para as autoras desta revisão, a busca dos pesquisadores por dados mais fidedignos sobre a incidência da violência e suas consequências. Krug, et al, 2002, referem-se a importância da boa coleta e análise dos dados para que se tenha ações mais eficazes na prevenção da violência e cuidados de suas vítimas.

No entanto, para que esse cuidado seja efetivo, faz-se necessário o conhecimento sobre os fatores de risco para a 
vitimização de crianças e adolescentes pela violência. Em três artigos (n. 2, n. 9 e n. 13 ) os autores consideram como fatores de risco a idade, o sexo, a classe socioeconômica, geralmente a mais baixa, e a composição familiar. Algumas pesquisas também apontaram que uma vez que o sujeito sofre a agressão em um contexto, estará mais propenso a ser vítima novamente no mesmo ambiente ou em outros, sendo, portanto a vitimização um fator de risco para outras agressões (ASSIS; AVANCI; OLIVEIRA, 2009; AVANCI et. al., 2009, XIMENE; OLIVEIRA; ASSIS, 2009).

Em relação ao suicídio os estudos discorrem que os fatores de risco para a prática do ato suicida podem estar relacionados à violência interpessoal sofrida anteriormente, além de sentimentos negativos e saúde mental abalada por consequência da violência ou não. Em relação à idade e sexo, referem que as tentativas são mais comuns na adolescência do que na infância, e que apesar de predominarem no sexo feminino, são os homens que cometem mais o ato suicida. Os autores também relacionam como fatores de risco para o suicídio relações familiares frágeis, problemas no relacionamento amoroso e morte de pessoas queridas (WERNECK et al, 2006 e MARCONDES FILHO, 2002).

Como visto anteriormente, os sintomas mais citados foram os depressivos e ansiosos. Diante destes dados é de suma importância destacar que alguns comportamentos das crianças e adolescentes podem ser considerados tanto fatores preditores para a exposição à violência quanto fatores de proteção. Avanci, et. al. (2009, p. 391) dizem que o "isolamento característico do comportamento depressivo protege a criança da exposição da violência, em especial da que acontece na escola e na vida social". Ximenes, Oliveira e Assis (2009, p. 429) também discorrem sobre essa relação bidirecional dizendo que "problemas de comportamento podem ser tanto preditores para a exposição à violência quanto consequência desta exposição".

\section{CONSIDERAÇÕES FINAIS}

Esta pesquisa contribui para o conhecimento sobre as publicações acerca da violência contra crianças e adolescentes, seus fatores de risco e as implicações para a saúde mental das vítimas.

Conhecer mais sobre a violência e suas consequências à saúde mental das vítimas pode favorecer o planejamento de ações de prevenção e a identificação de casos, podendo diminuir a subnotificação da violência.

Observa-se que ainda há muito a se discutir sobre a violência e suas consequências para a população infantojuvenil. Primeiramente se faz necessário a conscientização sobre a real dimensão desta problemática, considerando que suas causas e efeitos têm origem em diversos contextos: social, ambiental, cultural e político, e, portanto exigem abordagens multissetoriais nos três níveis de governo. Somente assim, esta poderá ser vista de forma global, facilitando a elaboração de estratégias eficazes para os três níveis de prevenção.

Vale ressaltar também que parte dos artigos publicados em 2010 podem não ter sido incluída nesta revisão devido ao período de coleta de dados, finalizada em março de 2011, e ao período de indexação de alguns periódicos. Finalmente, infere-se que a escolha dos descritores desta pesquisa influenciou no seu resultado, pois podem não ter conseguido localizar todo o universo de artigos publicados sobre esta temática nas bases pesquisadas ou mesmo não serem os mais adequados para abranger completamente nossos objetivos.

BONTEMPO, K. S.; PEREIRA, A. R. Mental health of children and teens victims of violence: a critical review of the literature. Rev. Ter. Ocup. Univ. São Paulo, v. 23, n. 2, p. 130-136 maio/ ago. 2012.

\begin{abstract}
It is about a critical literature review that aimed to identify and analyze bibliographical production in Brazilian journals on the consequences of violence on the children and adolescents mental health who are victims of violence. It has been performed the search of the articles published between January 2002 and December 2010 in Scielo and Lilacs electronic databases. Data were analyzed and distributed into four groups: the researched articles, the methodological approach, the several types of violence and its risk factors, and violence and its consequences for adolescent children mental health. Fivetten articles were found. It was noted that most of the articles had the teens as the target population and that the studies were performed primarily in schools and health services; the consequences of violence for children and youth mental health were addressed in articles as they used the instrument to collect data or according to the behavior of this population; the symptoms most often reported by the authors are depressive behavior, anxiety, and antisocial behavior. We can see the lack of research that report interventions to prevent or treat the consequences of violence on the mental health of this population.
\end{abstract}

KEY WORDS: Adolescent, Child, Mental Health, Violence. 


\section{REFERÊNCIAS}

ASSIS, S. G; AVANCI, J. Q.; OLIVEIRA, R. V. C. Desigualdade socioeconômicas e saúde mental infantil. Rev. saúde pública, São Paulo, v. 43, supl. 1, p. 92-100, ago. 2009.

AVANCI, J. Q ; ASSIS, S. G ; OLIVEIRA, R. V. C; PIRES, T. Quando a convivência com a violência aproxima a criança do comportamento depressivo. Ciênc. saúde coletiva, Rio de Janeiro, v.14, n.2, p. 383-394, mar./abr 2009.

AVANCI, J. Q; ASSIS, S. G; OLIVEIRA, R. V. C; FERREIRA, R. M; PESCE, R. P. Fatores associados aos problemas de saúde mental em adolescentes. Psic.: Teor. e Pesq., Brasília, v.23, n.3, p.287-294, jul./set. 2007.

BARDIN, L. Análise de conteúdo. Tradução de Luís Antero Reto, Augusto Pinheiro: Edições 70, 2010. 281 p.

BENETTI, S. P. C; PIZETTA, A; SCHWARTZ, C. B; HASS, R. A; MELO, V. L. Problemas de saúde mental na adolescência: características familiares, eventos traumáticos e violência. PsicoUSF, Itatiba, v. 15, n. 3, p. 321-332, set./dez. 2010

BRASIL. Ministério da Saúde. Secretaria de Assistência à Saúde. Notificação de maus-tratos contra crianças e adolescentes pelos profissionais de saúde:

um passo a mais na cidadania em saúde. Brasília: MS, 2002. 49 p.

BRASIL. Ministério da Saúde. Secretaria de Atenção a Saúde e Secretaria de Vigilância em Saúde. Impacto da violência na saúde das crianças e adolescentes: Prevenção de violências e Promoção da Cultura de paz. Brasília: MS, 2008. 16 p.

GRANT, M. J; BOOTHT, A. Tipes of review. Health inf. libr. j, Oxford, v. 26, p. 91-108, 2009.

KRUG, E G; DAHLBERG, L. L; MERCY, J. A; ZWI, A. B; LOZANO, R. eds. World report on violence and health. Geneva, World Health Organization, 2002.

MARCONDES FILHO, W ; MEZZAROBA, L ; TURINI, C. A ;
KOIKE, A ; MOTOMATSU JUNIOR, A; et. al. Tentativas de suicídio por substâncias químicas na adolescência e juventude. Adolesc. Latinoam., Porto Alegre, v.3, n.2, p.0-0, nov. 2002.

OLIVEIRA, A. S; ANTONIO, P. S. Sentimentos do adolescente relacionados ao fenômeno bullying: possibilidades para a assistencia de enfermagem nesse contexto. Rev. eletrônica enferm, Goiânia, v. 8, n. 1, p. 30-41, 2006.

PAULA, C. S; VEDOVATO, M. S; BORDIN, I. A; BARROA, M. G. S. M; D'ANTINO, M. E. I. F; MERCADANTE, M. T. Saúde mental e violência entre estudantes da sexta série de um município paulista. Rev. saúde pública, São Paulo, v. 42, n. 3, p. 524-528, jun. 2008.

SOUSA, M. K. B; SANTANA, J. S. S. Atenção ao adolescente vítima de violência: participação de gestores municipais de saúde. Ciênc. Saúde coletiva, Rio de Janeiro, vol 14, n.2, p. 547-555, mar- abr. 2009.

TANAKA, O. U; RIBEIRO, E.L. Ações de saúde mental na atenção básica: caminho para ampliação da integralidade da atenção. Ciênc. saúde coletiva, Rio de Janeiro, v. 14, n. 2, p.477-486, mar./abr. 2009.

VITOLO, Y. L. C; FLEITLICH-BILYK, B; GOODMAN, R; BORDIN, I. A. S. Crenças e atitudes educativas dos pais e problemas de saúde mental em escolares. Rev. saúde pública, São Paulo, v.39, n.5, p. 716-724, 2005.

WERNECK, G. L; HASSELMANN, M. H; PHEBO, L. B; VIEIRA, D. E; GOMES, V. L. O. Tentativas de suicídio em um hospital geral no Rio de Janeiro, Brasil. Cad. Saúde Pública, São Paulo, v. 22, n. 10, p. 2201-2206, out. 2006

XIMENES, L. F; OLIVEIRA, R. V. C.; ASSIS, S. G. Violência e transtorno de estresse pós-traumático na infância. Ciênc. saúde coletiva, Rio de Janeiro, v.14, n.2, p.417-433, mar./abr. 2009. 